University of Nebraska - Lincoln

DigitalCommons@University of Nebraska - Lincoln

State-Space Discretization of the Kalinin-Milyukov-Nash Cascade in a Sample-Data System Framework for Streamflow Forecasting

Jozsef Szilagyi

University of Nebraska-Lincoln, jszilagyi1@unl.edu

Follow this and additional works at: https://digitalcommons.unl.edu/natrespapers

Part of the Natural Resources and Conservation Commons, Natural Resources Management and Policy Commons, and the Other Environmental Sciences Commons

Szilagyi, Jozsef, "State-Space Discretization of the Kalinin-Milyukov-Nash Cascade in a Sample-Data System Framework for Streamflow Forecasting" (2003). Papers in Natural Resources. 889.

https://digitalcommons.unl.edu/natrespapers/889

This Article is brought to you for free and open access by the Natural Resources, School of at DigitalCommons@University of Nebraska - Lincoln. It has been accepted for inclusion in Papers in Natural Resources by an authorized administrator of DigitalCommons@University of Nebraska - Lincoln. 


\title{
State-Space Discretization of the Kalinin-Milyukov-Nash- Cascade in a Sample-Data System Framework for Streamflow Forecasting
}

\author{
Jozsef Szilagyi ${ }^{1}$
}

\begin{abstract}
A discretization of the continuous Kalinin-Milyukov-Nash-cascade is performed with the help of state-space analysis for hydrological forecasting of streamflow. A sample-data system approach is used during the discretization and results in discretely coincident values with the continuous model. The sample-data system uses input values measured instantly in time and assumes linear changes in the value of the input variable between discrete data measurements. Such description gives a generalized formulation of the pulse-data system approach often used in system engineering and discrete-time analysis of hydrological systems. An example is given to demonstrate that the approach results in improved forecasts of stream-discharge values when compared with the more traditional pulse-data system approach.
\end{abstract}

DOI: 10.1061/(ASCE)1084-0699(2003)8:6(339)

CE Database subject headings: Streamflow; Forecasting; Hydrologic models; Measurement; Hydrologic data.

\section{Introduction}

Hydrological processes, such as streamflow, are rarely measured continuously in time, and even in the rare events when they are (e.g., by the application of pressure-transducers), these measurements are subsequently discretized so the resulting discrete values can be stored and processed on a digital computer. A sample-data system is formed when the value of a variable assigned to discrete points in time corresponds to the instantaneous value of the same variable - whose value changes smoothly in time-at those discrete time-points. A good example for such a variable is streamflow which is measured (generally via stage-measurements using a rating-curve method) instantly in time. Precipitation, for example, is different because one rarely needs instantaneous values of precipitation intensities; rather one almost exclusively uses accumulated precipitation values of different durations which results in a series of step functions.

Working with discrete data has its consequences because hydrological processes, like most other natural phenomena, are continuous in time and the models describing them are continuous as well. As a consequence, discretized forms of these continuous models are often required to be formulated to become compatible with the discrete nature of the data available (Sherman 1932). The state-space approach of model discretization may be preferred when the nature of the application requires the handling of model uncertainties that may arise from measurement errors or inadequacies in model selection. In the case of operative hydrological

\footnotetext{
${ }^{1}$ Research Hydrologist/Assistant Professor, Conservation and Survey Division, Univ. of Nebraska-Lincoln, Lincoln, NE 68588-0517. E-mail:jszilagyi1@unl.edu

Note. Discussion open until April 1, 2004. Separate discussions must be submitted for individual papers. To extend the closing date by one month, a written request must be filed with the ASCE Managing Editor. The manuscript for this paper was submitted for review and possible publication on October 1, 2002; approved on March 3, 2003. This paper is part of the Journal of Hydrologic Engineering, Vol. 8, No. 6, November 1, 2003. (CASCE, ISSN 1084-0699/2003/6-339-347/\$18.00.
}

forecasting, the correct value of the forecasted river stage during floods is critical because a difference of a few percent in the value may mean the evacuation of densely populated areas. Under such circumstances, one strives to reduce any possible model uncertainty which, as of today, is commonly achieved by the application of certain digital filters, the most notable one having been developed by Kalman (1960) in a state-space framework.

The Kalinin-Milyukov-Nash (KMN)-cascade originally formulated by Nash (1957) for rainfall-runoff modeling, and independently of him by Kalinin and Milyukov (1957) for flood routing, assumes that the constant exponents $\left(m_{1}\right.$ and $\left.m_{2}\right)$ of the rating curve and that of storage $(S)$ as a function of stage $(y)$ are equal

$$
\begin{aligned}
& Q(t)=c_{1}[y(t)]^{m_{1}} \\
& S(t)=c_{2}[y(t)]^{m_{2}}
\end{aligned}
$$

for a characteristic reach of the stream, where $Q$ $=$ stream-discharge; $t=$ time; and $c_{1}$ and $c_{2}=$ constants. Eqs. $(1 a)$ and $(1 b)$ yield the linear storage equation $Q(t)=k S(t)$, where the inverse of $k$ is the so-called storage coefficient $(K)$ with a dimension of time. The storage equation combined with the lumped version of the continuity equation, $d S / d t=Q_{\text {in }}-Q_{\text {out }}$, for the characteristic reach transform into a first-order, constant coefficient, linear ordinary differential equation

$$
\frac{d S(t)}{d t}=-k S(t)+Q_{\text {in }}(t)
$$

The linearity condition ensures that the outflow of the characteristic reach through time can be calculated by the convolution integral of the inflow and the impulse response function $(h)$ [which in hydrology is called the instantaneous unit hydrograph (IUH)]

$$
Q_{\text {out }}(t)=\int_{0}^{t} h(\tau) Q_{\text {in }}(t-\tau) d \tau=\int_{0}^{t} h(t-\tau) Q_{\text {in }}(\tau) d \tau
$$

Assuming that a given stream section can be regarded as a series 
of $n$ characteristic reaches (where $n$ is an integer number) with identical storage coefficients, one obtains the well-known impulse-response function of the continuous $\mathrm{KMN}$-cascade

$$
h(t)=k \frac{(k t)^{n-1}}{(n-1) !} e^{-k t}
$$

By definition of the characteristic reach one can call such a reach a linear reservoir where the stored water in the reservoir is directly proportional to the outflow from it. Noticing that the output of the $i$ th reservoir is the input to the $(i+1)$ th reservoir, Eq. (2) can be written in matrix form for a cascade of order $n$ as (e.g., Szöllósi-Nagy 1982)

$$
\left[\begin{array}{c}
\dot{S}_{1}(t) \\
\dot{S}_{2}(t) \\
\dot{S}_{3}(t) \\
\vdots \\
\dot{S}_{n}(t)
\end{array}\right]=\left[\begin{array}{ccccc}
-k & & & & 0 \\
k & -k & & & \\
& k & -k & & \\
& & \ddots & \ddots & \\
0 & & & k & -k
\end{array}\right]\left[\begin{array}{c}
S_{1}(t) \\
S_{2}(t) \\
S_{3}(t) \\
\vdots \\
S_{n}(t)
\end{array}\right]+\left[\begin{array}{c}
1 \\
0 \\
0 \\
\vdots \\
0
\end{array}\right] Q_{\text {in }}(t)
$$

where the dot denotes the temporal rate of change in the variable and the subscripts denote the place of the reservoir in the line of cascade. Eq. (5) is the state equation of the continuous KMNcascade, and can be written in a more succinct form as

$$
\underline{\dot{S}}(t)=\underline{F} \underline{S}(t)+\underline{G} Q_{\text {in }}(t)
$$

where the state matrix $\underset{\underline{F}}{F}$ is an $n \times n$ Toeplitz matrix (SzöllósiNagy 1982; Nikolski 2002). The outflow from the last reservoir is the output of the whole system which can be written as

$$
Q_{\text {out }}(t)=[0,0,0, \ldots, k]\left[\begin{array}{c}
S_{1}(t) \\
S_{2}(t) \\
S_{3}(t) \\
\vdots \\
S_{n}(t)
\end{array}\right]=\underline{H} \underline{S}(t)
$$

The solution of the state equation (6) is given by (Szöllósi-Nagy 1982)

$$
\underline{S}(t)=\underline{\underline{\Phi}}\left(t, t_{0}\right) \underline{S}\left(t_{0}\right)+\int_{t_{0}}^{t} \Phi(t, \tau) \underline{G}(\tau) Q_{\mathrm{in}}(\tau) d \tau
$$

where for the KMN-cascade the input $(\underline{G})$ and output $(\underline{H})$ vectors are constant vectors, so is the system matrix, $F$, which causes the $n \times n$ state transition matrix, $\Phi$ depend only on the time-lag between $t$ and $t_{0} \cdot \Phi$ is the matrix exponential of $\underline{\underline{F}}$ such as

$$
\Phi\left(t, t_{0}\right)=e^{\underline{F} \cdot\left(t-t_{0}\right)}
$$

The elements of $\Phi$ are the following:

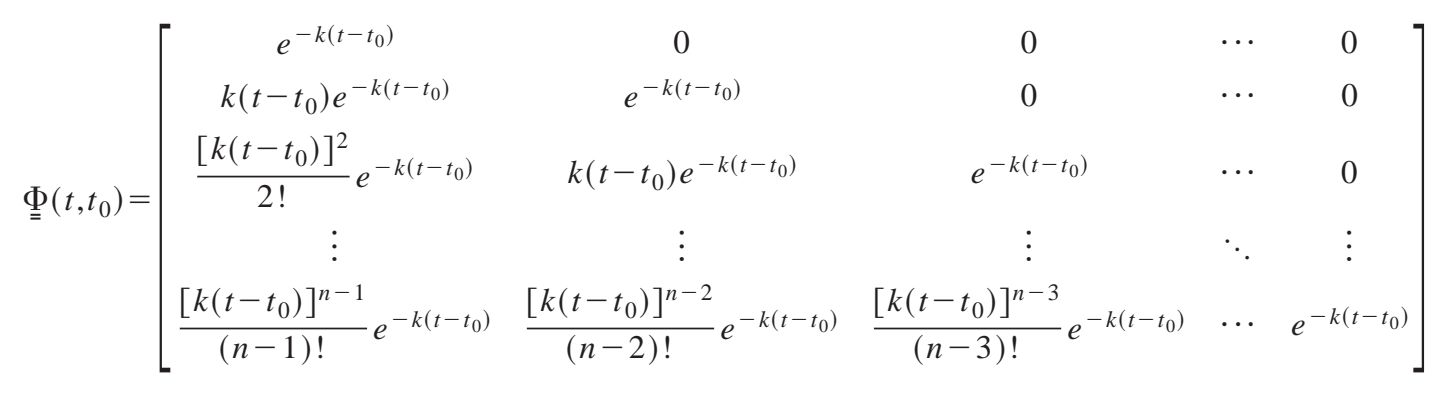

See Szöllosi-Nagy (1982) for the steps involved with the derivation of Eq. (10), where the Toeplitzian property of the state matrix was exploited. Combining Eqs. (7) and (8) and assuming that the system is initially relaxed, $\underline{S}\left(t_{0}\right)=0$ at $t_{0}=0$, i.e., the reservoirs are empty, one obtains

$$
Q_{\text {out }}(t)=\int_{0}^{t} \underline{H} \Phi(t, \tau) \underline{G} Q_{\text {in }}(\tau) d \tau=\int_{0}^{t} h(t-\tau) Q_{\text {in }}(\tau) d \tau
$$

which is the convolution equation (3) in matrix form. Note that the left-multiplication of $\Phi$ by $\underline{H}$ in Eq. (11) produces the last line of $\Phi$ as a row vector multiplied by $k$, and which upon further multiplication from right by $G$ results in the bottom left-most element of $\Phi$ (multiplied by $k$ ), thus recovering Eq. (4) for the IUH of the KMN-cascade.

Below it will be shown how the continuous state equation can be discretized first (1) in a pulse-data system framework following Szöllósi-Nagy $(1982,1989)$ and then (2) in the more general sample-data system framework which is the main contribution of the present study.

\section{Discretization of the Continuous State Equation of the Kalinin-Milyukov-Nash-Cascade in a Pulse-Data System Framework}

When the instantaneous value of $Q_{\text {in }}$ is available at discrete time intervals $(t=\Delta t, 2 \Delta t, 3 \Delta t, \ldots)$ of equal length, and the state variable $\underline{S}$ is known at time $t$ then, by virtue of Eq. (8), $S$ at time $t$ $+\Delta t$ can be calculated as (Szöllósi-Nagy 1989)

$$
\underline{S}(t+\Delta t)=\underline{\underline{\Phi}}(t+\Delta t, t) \underline{S}(t)+\int_{t}^{t+\Delta t} \underline{\underline{\Phi}}(t+\Delta t, \tau) \underline{G} Q_{\text {in }}(\tau) d \tau
$$

which transforms into the following simpler form provided that $Q_{\text {in }}(t)$ is taken to be constant at the value it obtains at time $t$, in the $[t, t+\Delta t)$ interval (Szöllosi-Nagy 1982)

$$
\underline{S}(t+\Delta t)=\underline{\Phi}(\Delta t) \underline{S}(t)+\underline{\Gamma}(\Delta t) Q_{\text {in }}(t)
$$

From Eq. (10) we obtain 


$$
\Phi(\Delta t)=\left[\begin{array}{ccccc}
e^{-k(\Delta t)} & 0 & 0 & \cdots & 0 \\
k(\Delta t) e^{-k(\Delta t)} & e^{-k(\Delta t)} & 0 & \cdots & 0 \\
\frac{[k(\Delta t)]^{2}}{2 !} e^{-k(\Delta t)} & k(\Delta t) e^{-k(\Delta t)} & e^{-k(\Delta t)} & \cdots & 0 \\
\vdots & \vdots & \vdots & \ddots & \vdots \\
\frac{[k(\Delta t)]^{n-1}}{(n-1) !} e^{-k(\Delta t)} & \frac{[k(\Delta t)]^{n-2}}{(n-2) !} e^{-k(\Delta t)} & \frac{[k(\Delta t)]^{n-3}}{(n-3) !} e^{-k(\Delta t)} & \cdots & e^{-k(\Delta t)}
\end{array}\right]
$$

where again the time invariance of the $F$ system matrix was utilized. The $\underline{\Gamma}$ vector results from Eq. (12) if the inflow value $Q_{\text {in }}$, which is assumed to be constant between the integral bounds, is brought outside the integral

$$
\underline{\Gamma}(\Delta t)=\int_{t}^{t+\Delta t} \Phi(t+\Delta t-\tau) \underline{\underline{G}} d \tau
$$

The $n \times 1 \underline{\Gamma}$ vector, which in a more general approach is indeed a matrix, is called the input transition matrix in system engineering. The $i$ th element or row of $\underline{\underline{T}}$ can be expressed as

$$
\begin{aligned}
\Gamma_{i}(\Delta t) & =\int_{t}^{t+\Delta t} \frac{k(t+\Delta t-\tau)^{i-1}}{(i-1) !} e^{-k(t+\Delta t-\tau)} d \tau \\
& =\frac{1}{k} \frac{1}{(i-1) !} \int_{0}^{k \Delta t} x^{i-1} e^{-x} d x \\
& =\frac{1}{k} \frac{\Gamma(i, k \Delta t)}{(i-1) !} \\
& =\frac{1}{k} \frac{\Gamma(i, k \Delta t)}{\Gamma(i)}
\end{aligned}
$$

where the $k(t+\Delta t-\tau)$ term, which is never negative, was substituted by $x$ in the integral, and the gamma-function's property for integers $i$, that $\Gamma(i)$ is equal to $(i-1)$ ! was utilized. The numerator on the right-hand-side of Eq. (16) is the so-called incomplete gamma function.

The impulse-response function of the continuous KMNcascade is replaced by the unit-pulse response function in the discrete model, and can be obtained, using the pulse-data system approach, by the following recursion (Szöllósi-Nagy 1989):

$$
\begin{gathered}
\underline{S}(\Delta t)=\underline{\Phi}(\Delta t) \underline{S}(0)+\underline{\Gamma}(\Delta t) Q_{\text {in }}(0) \\
\underline{S}(2 \Delta t)=\underline{\Phi}(\Delta t) \underline{S}(\Delta t)+\underline{\Gamma}(\Delta t) Q_{\text {in }}(\Delta t)=\underline{\Phi}^{2}(\Delta t) \underline{S}(0) \\
+\underline{\Phi}(\Delta t) \underline{\Gamma}(\Delta t) Q_{\text {in }}(0)+\underline{\Gamma}(\Delta t) Q_{\text {in }}(\Delta t) \\
\vdots \\
\underline{S}(m \Delta t)=\underline{\Phi}^{m}(\Delta t) \underline{S}(0)+\sum_{i=0}^{m-1} \underline{\Phi}^{m-1-i}(\Delta t) \underline{\Gamma}(\Delta t) Q_{\text {in }}(i \Delta t)
\end{gathered}
$$

From Eq. (17) the outflow $Q_{\text {out }}$ of the cascade at $t=m \Delta t$ is

$$
\begin{aligned}
Q_{\mathrm{out}}(m \Delta t)= & \underline{H} \underline{S}(m \Delta t)=\underline{H} \underline{\underline{\Phi}}^{m}(\Delta t) \underline{S}(0) \\
& +\sum_{i=0}^{m-1} \underline{H} \underline{\underline{\Phi}}^{m-1-i}(\Delta t) \underline{\Gamma}(\Delta t) Q_{\mathrm{in}}(i \Delta t)
\end{aligned}
$$

which produces the unit-pulse response function with the $\underline{S}(0)$ $=0$ choice as

$$
h(m \Delta t)=\underline{H} \underline{\Phi}^{m-1}(\Delta t) \underline{\Gamma}(\Delta t)
$$

Eq. (19) is the inner product of the last line of $\underline{\underline{\Phi}}^{m-1}$ (multiplied by $k$ ) and the $\underline{\Gamma}$ vector

$$
h(m \Delta t)=e^{-k(m-1) \Delta t}\left[\sum_{i=1}^{n} \frac{[k(m-1) \Delta t]^{n-1}}{(n-i) !} \frac{\Gamma(i, k \Delta t)}{\Gamma(i)}\right]
$$

where special use was made of the state transition matrix's definition (Szöllősi-Nagy 1982)

$$
\Phi^{m}(\Delta t)=\left(e^{F \Delta t}\right)^{m}=e^{F m \Delta t}
$$

Szöllósi-Nagy (1989) proved that the discrete model, given by the matrix triplet $[\underline{\underline{\Phi}}(\Delta t), \underline{\Gamma}(\Delta t), \underline{H}]$ is an adequate representation of the continuous KMN-cascade model defined by $[\underline{F}, \underline{G}, \underline{H}]$. Adequacy here means that the discrete version of the continuous KMN-cascade (1) gives identical output values at discrete points of time with the continuous model, provided the continuous model receives the same pulsed data input (Nash 1959; O'Connor 1982), and (2) preserves continuity of the original model.

\section{Discretization of the Continuous State Equation of the Kalinin-Milyukov-Nash-Cascade in a Sample-Data System Framework}

When applying the KMN-cascade model for flood routing, both the input and output variables of the model are generally streamflow values for larger river networks. The only exception may be the first upstream gauging station in each subbasin where the flow values are modeled with precipitation as input to the model. At these locations, the application of the pulse-data system framework is well justified since precipitation is generally reported as a constant accumulated value over a given period. At the other gauging stations farther downstream, however, the adaptation of a sample-data system seems to be more adequate, since streamflow, as mentioned earlier, is measured instantaneously and changes continuously between subsequent measurements.

In the sample-data system framework derivation of the discrete model, it is assumed that streamflow changes linearly between any two measurements. With this assumption Eq. (12) can be written as 


$$
\begin{aligned}
\underline{S}(t+\Delta t)= & \underline{\underline{\Phi}}(\Delta t) \underline{S}(t)+\int_{t}^{t+\Delta t} \underline{\underline{\Phi}}(t+\Delta t-\tau) \underline{G} u(\tau) d \tau \\
= & \underline{\underline{\Phi}}(\Delta t) \underline{S}(t)+\int_{t}^{t+\Delta t} \underline{\Phi}(t+\Delta t-\tau) \underline{G} \\
& \times\left[u(t)+\frac{u(t+\Delta t)-u(t)}{\Delta t}(\tau-t)\right] d \tau
\end{aligned}
$$

where inflow $Q_{\text {in }}$ from now on will be denoted with $u$ for easier notation. Observe that the state transition matrix remains the same, but, as is shown below, not the input transition vector $\underline{\Gamma}$. See the Appendix for the steps involved with the derivation of the new solution, which becomes

$$
\underline{S}(t+\Delta t)=\underline{\Phi}(\Delta t) \underline{S}(t)+\underline{\Gamma}_{1}(\Delta t) u(t+\Delta t)-\underline{\Gamma}_{2}(\Delta t) u(t)
$$

where the elements of $\underline{\Gamma}_{1}$ are

$$
\underline{\Gamma}_{1}(\Delta t)=\left[\begin{array}{c}
\frac{1}{k} \frac{\Gamma(1, k \Delta t)}{\Gamma(1)}\left[1+\frac{e^{-k \Delta t}}{\Gamma(1, k \Delta t)}-\frac{1}{k \Delta t}\right] \\
\frac{1}{k} \frac{\Gamma(2, k \Delta t)}{\Gamma(2)}\left[1+\frac{(k \Delta t) e^{-k \Delta t}}{\Gamma(2, k \Delta t)}-\frac{2}{k \Delta t}\right] \\
\frac{1}{k} \frac{\Gamma(3, k \Delta t)}{\Gamma(3)}\left[1+\frac{(k \Delta t)^{2} e^{-k \Delta t}}{\Gamma(3, k \Delta t)}-\frac{3}{k \Delta t}\right] \\
\vdots \\
\frac{1}{k} \frac{\Gamma(n, k \Delta t)}{\Gamma(n)}\left[1+\frac{(k \Delta t)^{n-1} e^{-k \Delta t}}{\Gamma(n, k \Delta t)}-\frac{n}{k \Delta t}\right]
\end{array}\right]
$$

and the same for $\underline{\Gamma}_{2}$ can be written as

$$
\underline{\Gamma}_{2}(\Delta t)=\left[\begin{array}{c}
\frac{1}{k} \frac{\Gamma(1, k \Delta t)}{\Gamma(1)}\left[\frac{e^{-k \Delta t}}{\Gamma(1, k \Delta t)}-\frac{1}{k \Delta t}\right] \\
\frac{1}{k} \frac{\Gamma(2, k \Delta t)}{\Gamma(2)}\left[\frac{k \Delta t e^{-k \Delta t}}{\Gamma(2, k \Delta t)}-\frac{2}{k \Delta t}\right] \\
\frac{1}{k} \frac{\Gamma(3, k \Delta t)}{\Gamma(3)}\left[\frac{(k \Delta t)^{2} e^{-k \Delta t}}{\Gamma(3, k \Delta t)}-\frac{3}{k \Delta t}\right] \\
\vdots \\
\frac{1}{k} \frac{\Gamma(n, k \Delta t)}{\Gamma(n)}\left[\frac{(k \Delta t)^{n-1} e^{-k \Delta t}}{\Gamma(n, k \Delta t)}-\frac{n}{k \Delta t}\right]
\end{array}\right]
$$

With this result, Eq. (18) becomes

$$
\begin{aligned}
Q_{\text {out }}(m \Delta t)= & \underline{H} \underline{S}(m \Delta t)=\underline{H} \underline{\underline{\Phi}}^{m}(\Delta t) \underline{S}(0)+\sum_{i=0}^{m-1} \underline{H} \underline{\underline{\Phi}}^{m-1-i}(\Delta t) \\
& \times\left\{\underline{\Gamma}_{1}(\Delta t) u[(i+1) \Delta t]-\underline{\Gamma}_{2}(\Delta t) u(i \Delta t)\right\}
\end{aligned}
$$

which shows that the output at time $t$ is not only influenced by the input at $t-\Delta t$ but also at $t$. This seemingly is a new development when compared to the pulse-data system description, where the output at time $t$ does not seem to be influenced by the synchronous input, as is suggested by Eq. (18). This however is not quite so, and here is the explanation.

The two discrete models, given by $[\Phi(\Delta t), \underline{\underline{\Gamma}}(\Delta t), \underline{H}]$ and $\left[\Phi(\Delta t), \underline{\Gamma}_{1}(\Delta t), \underline{\Gamma}_{2}(\Delta t), \underline{H}\right]$ are equivalent if their unit-pulse response functions are identical (Desoer 1970). In the pulse-data system framework, the unit-pulse response function can be obtained from Eq. (18) by letting the input to be unity at $t=0$ (when the system is relaxed) and zero otherwise. This means only one nonzero value for the discrete input because in the pulse-data system the constant inputs are indeed interpreted on closed (from left) and open (from right) time intervals $[t, t+\Delta t$ ), as is correctly denoted here. Openness of the time interval from the right means that the constant value over the interval is not interpreted at time $t+\Delta t$, because the input signal jumps to a new value at that time instant and the input cannot have two values simultaneously in time. To the contrary, within the sample-data system framework there are no jumps involved in the input signal representation; thus the unit pulse must be described by two values of unity over the sampling interval $[t, t+\Delta t]$, over which the input variable is assumed to change linearly and which is closed from both sides now. Note that the derivation of the unit-pulse response in both frameworks requires an integration of the input signal over the $[t, t+\Delta t]$ interval for which the openness or closedness of the interval does not make any difference, since the value of the integral does not change over a single point.

The unit-pulse response function of the sample-data system is obtained by letting $u$ be unity at both $t=0$ and $t=\Delta t$, assuming the system is relaxed. Note that whenever the input value is constant over the sampling interval, i.e., holds its last sample value, the two separate input transition vectors, $\underline{\Gamma}_{1}$ and $\underline{\Gamma}_{2}$ collapse into $\underline{\Gamma}$, the input transition vector of the pulse-data system, which means that the unit pulse response functions of the two frameworks are identical and so the two models of $[\Phi(\Delta t), \underline{\Gamma}(\Delta t), \underline{H}]$ and $\left[\Phi(\Delta t), \underline{\Gamma}_{1}(\Delta t), \underline{\Gamma}_{2}(\Delta t), \underline{H}\right]$ are indeed equivalent. Consequently, the output at $t+\Delta t$ in both frameworks are influenced by the simultaneous input; however, this influence is hidden in the pulse-data system representation because of the openness of the sampling interval. Note that though the IUH is the same for both data frameworks, the way the output is calculated is somewhat different [i.e., compare Eqs. (18) and (26)] between the two frameworks.

The equivalency of the two models does not mean that they give identical flood routing results since the inputs are assumed to behave differently in the two approaches between subsequent samples. If however one assumes that the input does not change over the sampling interval in the sample-data system [i.e., $u[(i$ $+1) \Delta t]=u(i \Delta t)$ is used in Eq. (26)], then the pulse-data system outputs are recovered, meaning that the sample-data system is a more general approach which, as a special case, includes the pulse-data system description. Because of the equivalency of the two discrete models, discrete coincidence and continuity of the discrete model for the pulse-data framework will remain valid in the sample-data system framework as well. For a demonstration of the model's discrete coincidence with the continuous KMNcascade, see Fig. 1, where a piecewise-linear input signal was routed through the continuous and the discrete models, with $n$ $=1, \Delta t=1[\mathrm{~T}]$, and $k=0.1\left[\mathrm{~T}^{-1}\right]$.

\section{Demonstration of the Discretized Kalinin-Milyukov- Nash-Cascade for Streamflow Forecasting}

Application of Eq. (26) in hydrological forecasting problems allows the user to choose between data systems without the need of changing the model structure. For pulsed data, such as accumulated precipitation, $u$ at $(i+1) \Delta t$ in Eq. (26) is taken equal to the value of $u$ at $i \Delta t$. This may generally be required, when precipitation is transformed into effective precipitation to be used as input when making streamflow predictions for the first gauging station along the stream. For other gauging stations downstream the inputs will be streamflow values in the flow routing between any two gauging stations requiring a sample-data system ap- 


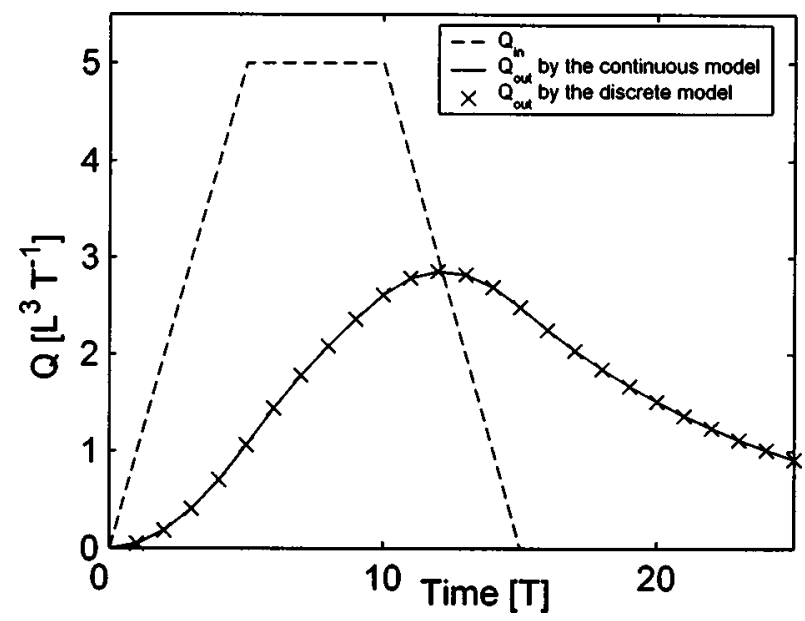

Fig. 1. Routing of piecewise-linear input with continuous and discrete models using sample-data system approach. Here, $n=1, \Delta t$ $=1[\mathrm{~T}]$, and $k=0.1\left[\mathrm{~T}^{-1}\right]$.

proach since streamflow values are measured instantaneously and change smoothly rather than through jumps.

When applying Eq. (26) to forecast streamflow at a downstream location using stream-flow information upstream, the value of $u$ at time $t+\Delta t$, the time of the forecast, is not yet known. A discrete and empirical version of Taylor's expansion may be used to estimate $u$ at time $t+i \Delta t(i=1,2,3)$, which can be written as

$$
\hat{u}(t+i \Delta t)=u(t)+\left(\sum_{j=1}^{i} c^{j}\right)[u(t)-u(t-\Delta t)]
$$

if one stops after the first-order term in the expansion employing a backward-difference scheme and assuming equidistant sampling; and the same becomes

$$
\begin{aligned}
\hat{u}(t+i \Delta t)= & u(t)+\left(\sum_{j=1}^{i} c^{j}\right)\{u(t)-u(t-\Delta t) \\
& +0.5[u(t)-2 u(t-\Delta t)+u(t-2 \Delta t)]\}
\end{aligned}
$$

when using a second-order expansion. The empirical constant $c$ in the expressions accounts for the random nature of the signal and must be optimized for best results in the estimation. Note that Eqs. (27) and (28) can be considered as a special, primitive form of an autoregressive process. The advantage of these equations is that they contain only one parameter to be optimized. Note also that the way future input is estimated in our demonstration is not the focus of this study. These estimates are generally available as operative model forecasts for the upstream gauges using inputs even further upstream, when performing this kind of nested-type predictions.

Before forecasting, one must estimate the state variable, $\underline{S}(0)$ at the start of the calculations. This can be achieved by inverting Eqs. (18) and (26), respectively, provided they are written in a super-matrix structure where each line consists of Eqs. (18) and (26), respectively, with $m$ changing from 1 to $n$. The so-derived $n \times n$ matrix $\Omega=\left[\underline{H} \Phi, \underline{H} \Phi^{2}, \ldots, \underline{H} \Phi^{n}\right]^{\prime}$ where the prime denotes the transpose of the matrix, is the observability matrix of the discrete model, and is never singular provided $n \geqslant 1, k>0$, and $\Delta t>0$ (Szöllósi-Nagy 1987, 1989).

Stream stages are recorded at 6 a.m. each day for the Danube in Hungary. The stage measurements are transformed into streamflow using a rating-curve method. In our demonstration of the discrete model, streamflow at Baja, about $200 \mathrm{~km}$ downstream of
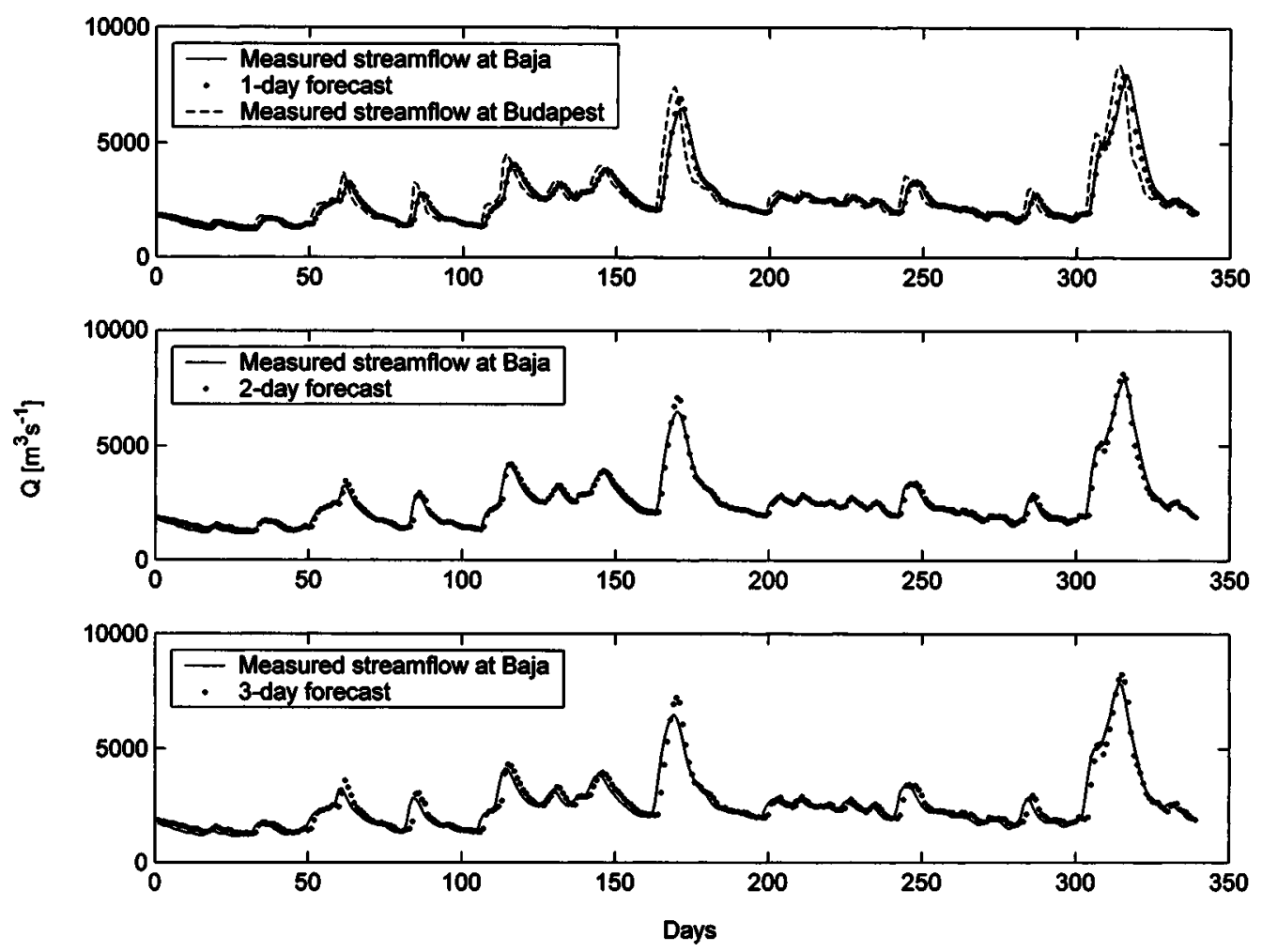

Fig. 2. Measured streamflow at Budapest and Baja (Danube) at 6 a.m. each day and 1-2-3-day forecasts for Baja using pulse-data system 
Table 1. Optimized Parameter Values and Model Performance Statistics of Discrete Model for All Three Lead-Times Combined

\begin{tabular}{lcc}
\hline Forecasts & $\begin{array}{c}\text { Pulse-data } \\
\text { system }\end{array}$ & $\begin{array}{c}\text { Sample-data } \\
\text { system }\end{array}$ \\
\hline With no input forecasts & 1 & 1 \\
$\quad n_{\text {opt }}$ & 0.6 & 0.4 \\
$k_{\text {opt }}\left[\mathrm{d}^{-1}\right]$ & - & 0.8 \\
$c_{\text {opt }}$ (first order Taylor expansion $)$ & 423 & 379 \\
Mean root-square error $\left[\mathrm{m}^{3} \mathrm{~s}^{-1}\right]$ & 95.8 & 96.7 \\
Nash-Sutcliffe efficiency coefficient & & \\
& & \\
{$[\%]=100\left(1-\frac{\sum\left(\hat{Q}_{i}-Q_{i}\right)^{2}}{\sum\left(Q_{i}-m_{Q}\right)^{2}}\right)$} & & 2 \\
With perfect input forecasts & 2 & 0.9 \\
$n_{\text {opt }}$ & 1 & 262 \\
$k_{\text {opt }}\left[\mathrm{d}^{-1}\right]$ & 286 & 98.4 \\
Mean root-square error $\left[\mathrm{m}^{3} \mathrm{~s}^{-1}\right]$ & 98.1 & \\
Nash-Sutcliffe efficiency coefficient & & \\
$\quad[\%]=100\left(1-\frac{\sum\left(\hat{Q}_{i}-Q_{i}\right)^{2}}{\sum\left(Q_{i}-m_{Q}\right)^{2}}\right)$ &
\end{tabular}

Note: Here $m_{Q}=2,317 \mathrm{~m}^{3} \mathrm{~s}^{-1}$ is mean streamflow (sample size $=340$ ) at Baja. In first case scenario, future streamflow at $t+i \Delta t(i=1,2,3)$ is estimated with help of empirical Taylor's expansion for sample-data system at Budapest, while in pulse-date system it is taken equal to last measured value. In second scenario it was assumed that perfect streamflow forecasts were available at Budapest for $t+i \Delta t(i=1,2,3)$, which means measured streamflow at those days were also used as inputs in routing.
Budapest, is forecasted from measurements in Budapest with a lead time varying from one to three days. In the first experiment, a pulse-data system framework is used while in the second one a sample-data system approach is applied with the discrete model. In both cases the $n$ and $k$ values are optimized by systematically changing their values within a preset range and accuracy for each parameter when running the model repeatedly to find the smallest mean root-square errors in the estimates. With the sample-data system approach, the above first- and second-order empirical Taylor-expansions are applied to estimate $u$ at time $t+i \Delta t$ ( $i$ $=1,2,3)$ from measured data at $t$, with $c$ being a third parameter to be optimized. The model was run without being continuously updated, which means the recalculation of $\underline{S}(0)$ for each day before the new forecast is issued, a common practice in operational forecasting to ensure best possible model results. The objective here is only to show that the model can be run in a pulse and also in a sample-data system approach without changing anything in the model structure and also to verify whether a sampledata system approach improves the forecasts or not, without going into details of how to manage errors in the forecasts, which can be done with the aforementioned application of the Kalmanfilter or by a simple autoregressive model component (Ahsan and O’Connor 1994).

Fig. 2 displays the one-, two-, and three-day forecasts for Baja using a pulse-data system approach. Note that the last flood event happened to be a record flood at Budapest and was part of the mayhem Europe experienced in the summer of 2002 with several casualties and flood damages in billions of dollars across the continent. The model predicted the record flood rather accurately with a $24 \mathrm{~h}$ lead-time, but it was less accurate with the second largest flood within the display period of the figure. Optimization resulted in $n=1$, and $k=0.6 \mathrm{~d}^{-1}$. See Table 1 for the error statis-
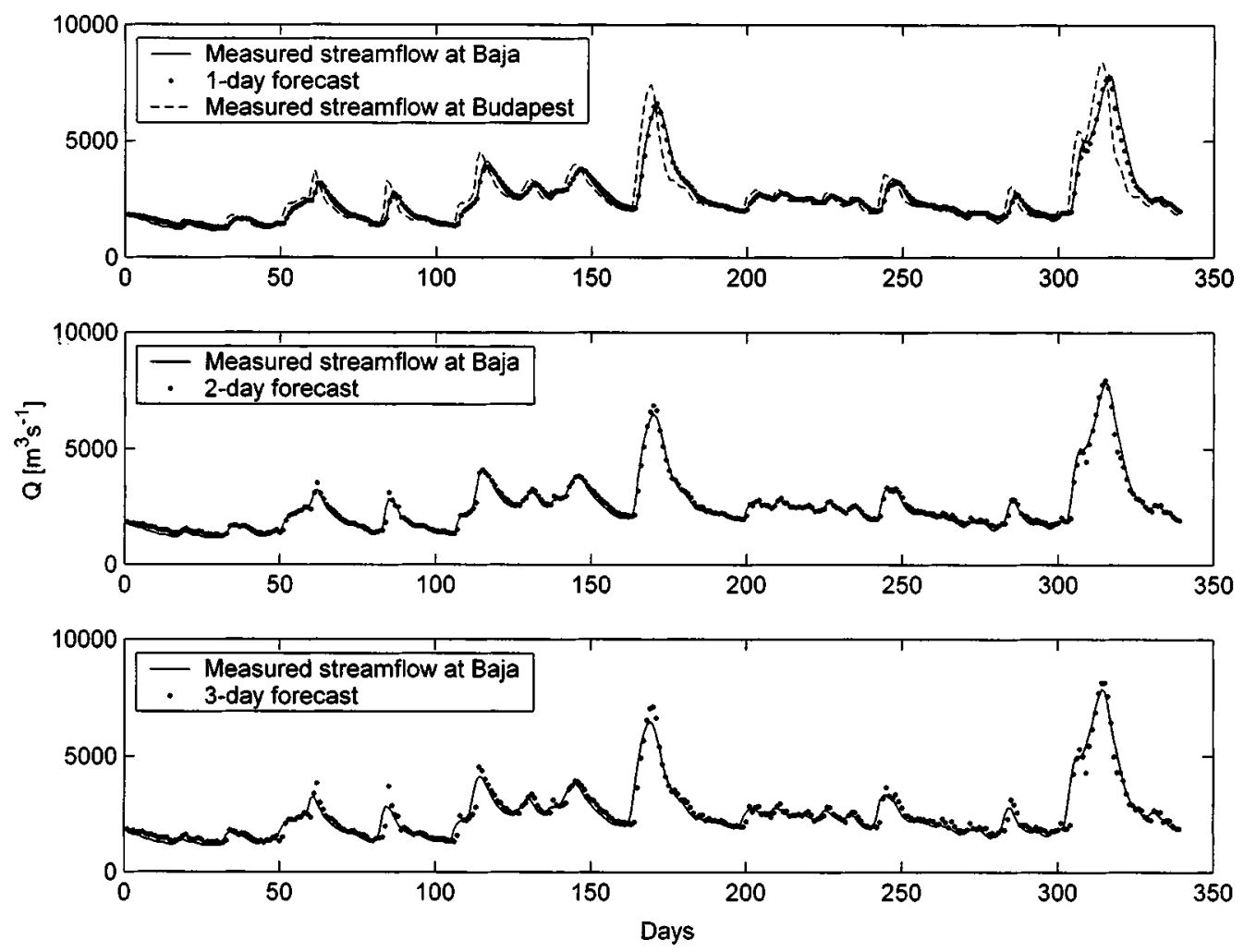

Fig. 3. Measured streamflow at Budapest and Baja (Danube) at 6 a.m. each day and 1-2-3-day forecasts for Baja using sample-data system. Temporal extrapolation of inflow at Budapest involved a first-order Taylor's expansion in discrete time. 

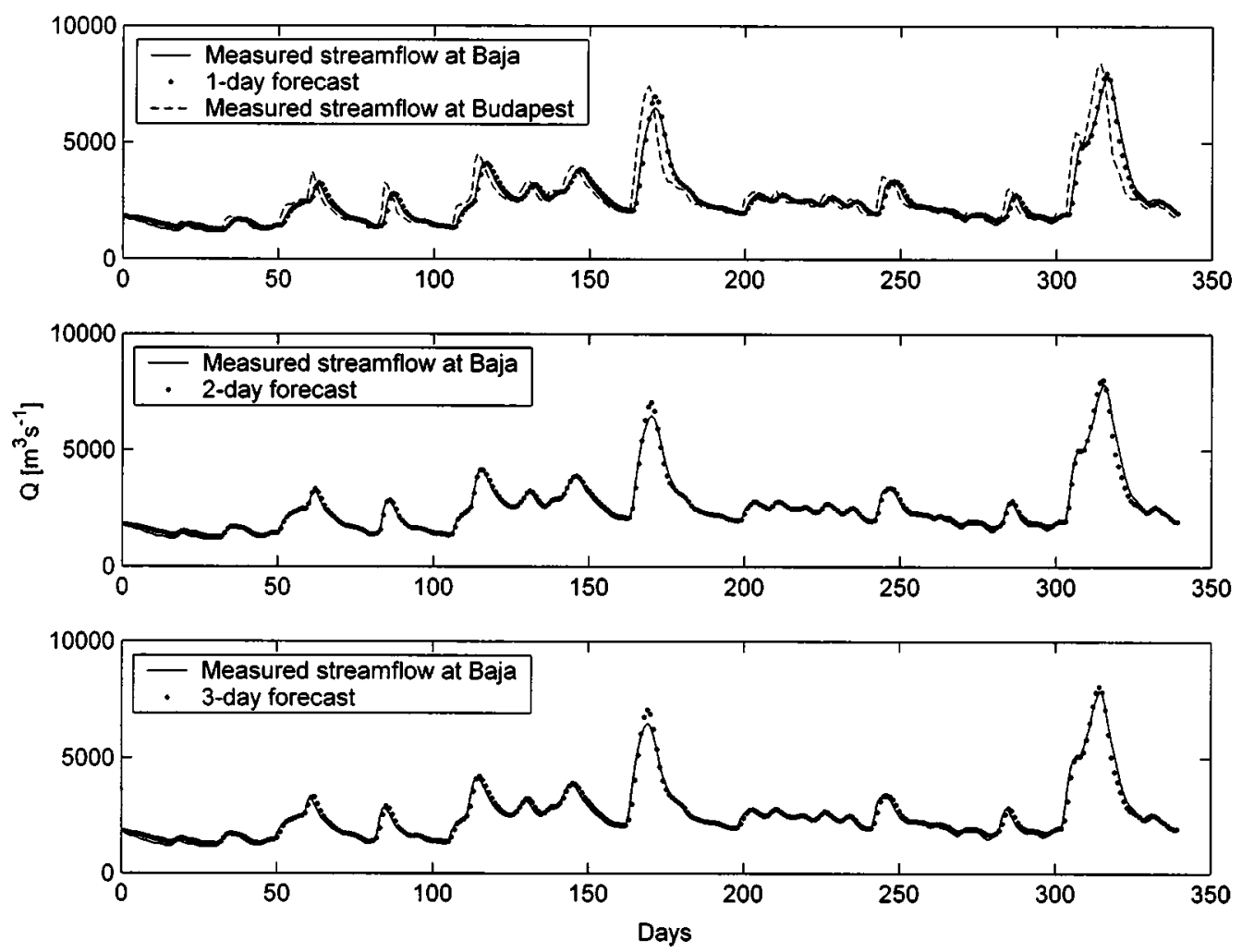

Fig. 4. Measured streamflow at Budapest and Baja (Danube) at 6 a.m. each day and 1-2-3-day forecasts for Baja using pulse-data system. For two- and three-day forecasts, measured streamflow on target day minus one at Budapest, simulating a perfect inflow forecast scenario, was used.
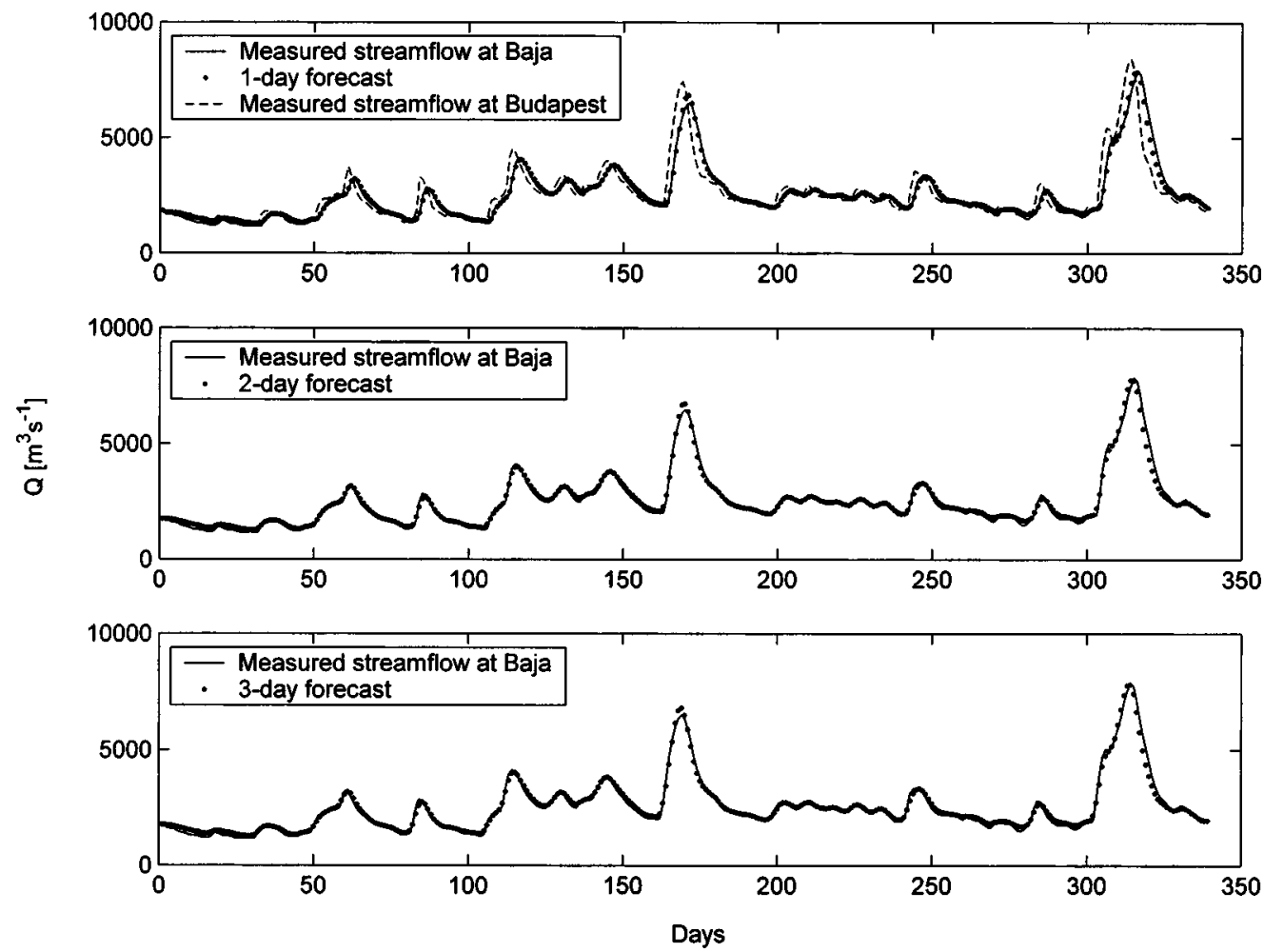

Fig. 5. Measured streamflow at Budapest and Baja (Danube) at 6 a.m. each day and 1-2-3-day forecasts for Baja using sample-data system. For each forecast, measured streamflow on target day minus one and on target day at Budapest, simulating perfect inflow forecast scenario, was used. 
tics. Fig. 3 displays the forecasts when a sample-data system approach is used with optimized values of $n=1, k=0.4 \mathrm{~d}^{-1}$, and $c=0.8$ using a first-order empirical Taylor expansion for estimating input streamflow at future times. There is not much improvement in predicting the record flood, but clearly there is improvement with the second largest flood of the figure over the pulsedata system approach. By switching from a pulse- to sample-data system, the mean root-square error (MRSE), combined for the three lead times, decreased by $10 \%$, while the already high NashSutcliffe efficiency coefficient (NSEC, see Table 1) increased by $1 \%$ for the combined forecasts.

In the second set of experiments, (see Figs. 4 and 5), forecasts for the input values at Budapest were also included in the routing. This again is routine practice with hydrological forecasting services since a good forecast for the upstream station using flow information one station further upstream can significantly improve forecasts from the given station to a downstream one. It was assumed here, as an extreme, that these forecasts are perfect, i.e., they yield in the exact measured values for future days. This way the effect of upstream prediction errors on the behavior of the forecasts at Baja within the two data system frameworks could be eliminated. See Table 1 for the new optimized values of $n$ and $k$, which shows that the inclusion of forecasted inputs even affect the optimum value of the model parameters. The new MRSE is again improved by $9 \%$ when switching from the pulsedata system to sample-data system. The NSEC value however is so high now even with the pulse-data system that it can change only very little $(0.3 \%)$ by switching between the two data systems.

In summary, it can be stated that the sample-data system approach seems to be better suited for streamflow routing than the pulse-data system framework because it can account for the continuously changing streamflow values more efficiently than the latter one. Both systems use the same kind of information with the only difference being that the pulse-data system assumes no change in the input value during the sample interval, while the sample-data system assumes a linear change. The two data systems can be used with the same state-space model formulation provided the model is formulated using the sample-data framework, which this way is a natural generalization of the pulse-data system.

\section{Acknowledgments}

The writer is grateful to Charles Flowerday, Gabor Balint, and to two anonymous reviewers for their valuable comments on an earlier version of the manuscript.

\section{Appendix}

In the derivation of Eq. (23) one must start from Eq. (22). For clarity of writing, the steps involved with the integration will be demonstrated on the $i$ th element of the vector-valued integrand. The $i$ th element of $\underline{S}(t+\Delta t)$ in Eq. (22), starting with an initially relaxed system at time $t$ can be expressed as

$$
\begin{aligned}
\int_{t}^{t+\Delta t} & \Phi_{i, 1}(t+\Delta t-\tau) u(\tau) d \tau \\
= & \int_{t}^{t+\Delta t} \Phi_{i, 1}(t+\Delta t-\tau)\left[u(t)+\frac{u(t+\Delta t)-u(t)}{\Delta t}(\tau-t)\right] d \tau \\
= & \int_{t}^{t+\Delta t}\left[\Phi_{i, 1}(t+\Delta t-\tau) u(t)+\Phi_{i, 1}(t+\Delta t-\tau)\right.
\end{aligned}
$$

$$
\left.\times \frac{u(t+\Delta t)-u(t)}{\Delta t} \tau-\Phi_{i, 1}(t+\Delta t-\tau) \frac{u(t+\Delta t)-u(t)}{\Delta t} t\right] d \tau
$$

If one performs a change of variables as $\tau^{*}=k(t+\Delta t-\tau)$, then the first term of the integral transforms into

$$
\begin{aligned}
u(t) \frac{1}{k} \frac{1}{(i-1) !} \int_{0}^{k \Delta t} \frac{\tau^{*(i-1)}}{e^{\tau^{*}}} d \tau^{*} & =u(t) \frac{1}{k} \frac{1}{(i-1) !} \Gamma(i, k \Delta t) \\
& =u(t) \frac{1}{k} \frac{\Gamma(i, k \Delta t)}{\Gamma(i)}
\end{aligned}
$$

where $\Phi_{i, l}$ was used from Eq. (14). Similarly, the third term of Eq. (29) will yield

$$
\frac{t}{k} \frac{u(t)-u(t+\Delta t)}{\Delta t} \frac{\Gamma(i, k \Delta t)}{\Gamma(i)}
$$

whereas the second term becomes

$$
\begin{aligned}
\frac{1}{k} \frac{1}{(i-1) !} \frac{u(t+\Delta t)-u(t)}{\Delta t} \int_{0}^{k \Delta t} \frac{\tau^{*(i-1)}}{e^{\tau^{*}}}\left(t+\Delta t-\frac{1}{k} \tau^{*}\right) d \tau^{*} \\
\left.=\frac{u(t+\Delta t)-u(t)\left[\frac{1}{k} \frac{\Gamma(i, k \Delta t)}{\Gamma(i)}(t+\Delta t)\right.}{\Delta t}-\frac{1}{k^{2}} \frac{1}{(i-1) !} \int_{0}^{k \Delta t} \frac{\tau^{*(i-1)}}{e^{\tau^{*}}} \tau^{*} d \tau^{*}\right] \\
=\frac{u(t+\Delta t)-u(t)}{\Delta t}\left[\frac{t+\Delta t}{k} \frac{\Gamma(i, k \Delta t)}{\Gamma(i)}-\frac{1}{k^{2}} \frac{\Gamma(i+1, k \Delta t)}{\Gamma(i)}\right] \\
=\frac{u(t+\Delta t)-u(t)}{\Delta t}\left[\frac{t+\Delta t}{k} \frac{\Gamma(i, k \Delta t)}{\Gamma(i)}\right. \\
\left.\quad-\frac{1}{k^{2}} \frac{\left.i \Gamma(i, k \Delta t)-(k \Delta t)^{i} e^{-k \Delta t}\right]}{\Gamma(i)}\right]
\end{aligned}
$$

where the algebraic identity $\Gamma(a+1, x)=a \Gamma(a, x)-x^{a} e^{-x}$ was used (Abramowitz and Stegun 1965) and where $\Gamma(a, x)$ $=\int_{0}^{x} e^{-t} t^{a-1} d t$. After combining all three terms one obtains

$$
\begin{aligned}
\int_{t}^{t+\Delta t} \Phi_{i, 1}(t+\Delta t-\tau) u(\tau) d \tau= & \frac{1}{k} \frac{\Gamma(i, k \Delta t)}{\Gamma(i)}\left[\left[1+\Lambda_{i}(\Delta t)\right]\right. \\
& \left.\times u(t+\Delta t)-\Lambda_{i}(\Delta t) u(t)\right]
\end{aligned}
$$

with $\Lambda_{i}(\Delta t)$ being

$$
\Lambda_{i}(\Delta t)=\frac{(k \Delta t)^{i-1} e^{-k \Delta t}}{\Gamma(i, k \Delta t)}-\frac{i}{k \Delta t}
$$

Eq. (23) results by defining $\Gamma_{1}^{i}(\Delta t)$ as

$$
\Gamma_{1}^{i}(\Delta t)=\frac{1}{k} \frac{\Gamma(i, k \Delta t)}{\Gamma(i)}\left[1+\Lambda_{i}(\Delta t)\right]
$$

and similarly, $\Gamma_{2}^{i}(\Delta t)$ as

$$
\Gamma_{2}^{i}(\Delta t)=\frac{1}{k} \frac{\Gamma(i, k \Delta t)}{\Gamma(i)} \Lambda_{i}(\Delta t)
$$


where the superscript $i$ in the $\Gamma_{1}$ and $\Gamma_{2}$ function definitions denotes the $i$ th element in the $n \times 1$ vectors.

\section{References}

Abramowitz, M., and Stegun, I. A. (1965). Handbook of mathematical functions, Dover, New York.

Ahsan, M., and O'Connor, K. M. (1994). “A reappraisal of the Kalman filtering technique, as applied in river flow forecasting." J. Hydrol., 161, 197-226.

Desoer, C. A. (1970). Notes for a second course on linear systems, Van Nostrand, New York.

Kalinin, G. P., and Milyukov, P. I. (1957). "Raschete neustanovivshegosya dvizheniya vody $\mathrm{v}$ otkrytykh ruslakh (On the computation of unsteady flow in open channels)." Met. i Gydrologia Zhurnal, 10, 10-18 (Leningrad).

Kalman, R. E. (1960). "A new approach to linear filtering and prediction problems.” ASME J. Basic Eng., 82D, 35-45.
Nash, J. E. (1957). "The form of the instantaneous unit hydrograph." Int. Assoc. Sci. Hydrol. Publ., 45(3), 114-131.

Nash, J. E. (1959). "A note on the Muskingum flood routing methods." $J$. Geophys. Res., 64(8), 1053.

Nikolski, N. K. (2002). "Operators, functions, and systems: an easy reading.” Math. Surv. Monogr. Am. Math. Soc., 92-93.

O'Connor, K. M. (1982). "Derivation of discretely coincident forms of continuous linear time-invariant models using the transfer function approach." J. Hydrol., 59, 1-48.

Sherman, L. K. (1932). "Streamflow from rainfall by the unit-graph method." Eng. News-Rec., 108, 501-505.

Szöllosi-Nagy, A. (1982). "The discretization of the continuous linear cascade by means of state space analysis." J. Hydrol., 58, 223-236.

Szöllôsi-Nagy, A. (1987). "Input detection by the discrete linear cascade model." J. Hydrol., 89, 353-370.

Szöllősi-Nagy, A. (1989). Real-time stream-flow forecasting using dynamically structured deterministic-stochastic models (In Hungarian: A mederbeli lefolyás real-time előrejelzése dinamikus strukturálissztochasztikus modellekkel), Vizgazdálkodási Tudományos Kutató Központ (VITUKI), Budapest. 